\title{
The confinement of helium tokamak plasmas, impact of electron heating, turbulent transport and zonal flows
}

\author{
P. Manas, ${ }^{1}$ C. Angioni, ${ }^{1}$ A. Kappatou,${ }^{1}$ F. Ryter,${ }^{1}$ P. A. Schneider,${ }^{1}$ and the ASDEX Upgrade Team ${ }^{2}$ \\ ${ }^{1}$ Max-Planck-Institut für Plasmaphysik, D-85748 Garching, Germany \\ ${ }^{2}$ See appendix of "A. Kallenbach et al., Nucl. Fusion 57 (2017) 102015"
}

(Dated: January 17, 2018)

\begin{abstract}
In tokamaks, helium plasmas are regularly observed to have significantly lower confinement than deuterium plasmas. For the first time, in the ASDEX Upgrade tokamak the confinement of helium plasmas is experimentally demonstrated to increase with increasing fraction of electron heating, reaching values comparable to those of the deuterium plasmas. Nonlinear gyrokinetic simulations show that the different impact of zonal flows in regulating the core turbulence in the limit of low electron heating in $\mathrm{D}$ and He plasmas breaks the gyro-Bohm scaling of transport, leading to higher levels of transport in He. The thermal coupling between electrons and ions and stronger destabilization of electron temperature gradient modes lead to reduced confinement at the edge of He plasmas.
\end{abstract}

PACS numbers:

Energy confinement times in helium tokamak plasmas have been regularly found lower compared to similar deuterium plasmas by approximately $\sim 30 \%[1,2]$. This observation is in contradiction with the gyro-Bohm scaling of the turbulent transport, according to which turbulent eddies responsible for radial transport are theoretically expected to scale with the Larmor radius $\rho=m_{i} v_{t h} / Z e B$ [3] with $m_{i}$ the ion mass, $v_{t h}=\sqrt{T / m_{i}}$ the thermal velocity, $Z$ the charge number, $e$ the elementary charge and $B$ the magnetic field strength. The breaking of the gyroBohm scaling is also routinely observed in experiments using isotopes of hydrogen (e.g. $[1,4,5])$ and is called the isotope effect. While many theoretical mechanisms were proposed to explain these observations such as global effects [6], external radial electric field shearing $(E \times B$ shearing) [7], and more recently via the coupling of zonal flows with electromagnetic effects and $E \times B$ shearing [8] or with collisionnal effects [9], no clear theoretical picture has been drawn yet. Aside from a practical operational characterisation of He plasmas confinement in tokamaks, which is also of high relevance for the prediction of the proposed non-nuclear phase of ITER operation, studies of such plasmas have direct implications regarding the isotope effect and could help narrowing down important theoretical ingredients.

The discharges presented here have been performed in the ASDEX Upgrade (AUG) tokamak. Deuterium and helium companion steady phases (identical parameters) in both $\mathrm{L}$ and $\mathrm{H}$-mode plasmas are compared. H-mode plasmas are produced at a plasma current of $I_{p}=0.6 \mathrm{MA}$ and toroidal magnetic field strength $B_{T}=2.5 \mathrm{~T}$ with the Electron Cyclotron Resonance Heating power $\left(P_{\mathrm{ECRH}}\right)$ ranging from 0.7 to $2.7 \mathrm{MW}$ and the neutral beam heating power from 1.4 to $6 \mathrm{MW}$. The line averaged core electron densities range from $n_{e}=4 \times 10^{19} \mathrm{~m}^{-3}$ to $n_{e}=6.2 \times 10^{19} \mathrm{~m}^{-3}$. Two pairs of L-mode plasmas have also been investigated at $I_{p}=1 \mathrm{MA}, B_{T}=2.5 \mathrm{~T}$,
$P_{\mathrm{ECRH}}=0.7 \mathrm{MW}$, with $n_{e}=2.1 \times 10^{19} \mathrm{~m}^{-3}$ and $n_{e}=4.5 \times 10^{19} \mathrm{~m}^{-3}$. Input powers, line averaged core electron densities and the magnetic field geometry were matched between helium and deuterium steady phases, allowing direct comparisons of the temperature profiles and thereby of the confinement. The Neutral Beam Injection (NBI) power deposition slightly differs in $\mathrm{He}$ and D. In He, hydrogen beams were used, yielding a reduced input torque compared to $\mathrm{D}$ beams and a total power evenly deposited on the ions and electrons whereas for D plasmas, D beams were used with $60 \%$ of the total power deposited on the ions. From the variety of heating schemes used in this study, it is interesting to compare how the total plasma stored energy $\left(W_{\mathrm{MHD}}\right)$ in He and D plasmas scales with the total heating power $\left(P_{\mathrm{TOT}}\right)$. In Fig. 1, this scaling is shown for H-modes only. Since the plasma densities are not exactly the same for all phases, the stored energy has been rescaled with the ITERH98y-2 [10] dependence of the confinement time on the line averaged core density $n_{e, 19}^{0.41}$ in units of $10^{19} \mathrm{~m}^{-3}$. Other exponents of the density dependence have been also tested, from different regressions, with no significant variation of the results.

In AUG deuterium $\mathrm{H}$-mode plasmas, the addition of ECRH to NBI heating does not affect confinement in the intermediate to high density range, consistently with $[11,12]$, while it reduces the confinement at low density [13]. Within the range of NBI powers used, the stored energy of D plasmas is larger than that of the corresponding He plasmas and, more importantly, has a more favorable dependence on $P_{\mathrm{TOT}}$. In contrast, in scenarios with significant ECRH heating, the plasma stored energy in helium has a more beneficial scaling. In the case of a larger fraction of ECRH and a lower electron density (green symbols in Fig. 1), the stored energy is practically identical in helium and deuterium plasmas.

The observation of different trends in the helium 


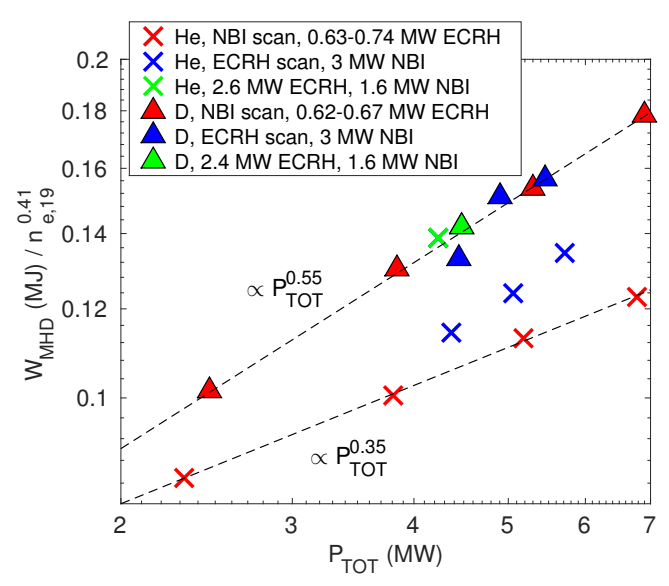

FIG. 1: Plasma stored energy (rescaled with the density scaling of ITERH98y-2) against the total heating power for H-mode helium and deuterium plasmas.

plasma stored energy with respect to NBI and ECRH heating powers is further highlighted in Fig. 2 where the ratio of the plasma stored energy in helium over deuterium is shown against the ratio of the ECRH power over the total power. The stored energies are computed in two different ways: from a function parametrization algorithm based on magnetic signals measurements ( $W_{\mathrm{MHD}}$ ) and from the measured electron and main ion kinetic profiles to disentangle the core and edge contributions to the total stored energies. The core contribution is computed using $\int_{0}^{c}[P-P(c)] \partial V / \partial r \mathrm{~d} r$ with $P$ the total kinetic pressure, $V$ the plasma volume and $r$ the minor radius. For $\mathrm{H}$-modes $c$ is chosen at the pedestal top with $r / a=0.85$, with $a$ the minor radius at the separatrix, yielding to a ratio $W_{\text {core }} / W_{\text {tot }}$ (with $W_{\text {tot }}$ calculated with $r / a=1)$ of $\sim 50 \%$ in $\mathrm{D}$ and $\sim 55 \%$ in He. The two Lmode plasmas are also included in this analysis. In these cases $c$ is chosen at $r / a=0.77$ to obtain the same ratios of $W_{\text {core }} / W_{\text {tot }}$ as in H-modes.

The stored energy (or equivalently the confinement time) in helium deteriorates compared to deuterium with high NBI heating and improves with high fractions of ECRH. The ratios of plasma stored energies in L-modes also follow the general tendency provided by the $\mathrm{H}$ modes. Core and edge contributions to the stored energies identify two different trends. In the core, the stored energy in He increases throughout the scan in $P_{\mathrm{ECRH}} / P_{\mathrm{TOT}}$ from $\sim 80 \%$ of that in $\mathrm{D}$ with strong NBI heating to $\sim 120 \%$ with strong ECRH and low density. In contrast, the edge stored energy in He is systematically lower compared to $\mathrm{D}$ for medium to high density plasmas whereas in the lowest density case, it becomes similar. This behaviour is also observed in L-modes where only the core line averaged densities differ between the two pairs of plasmas studied and where the core confinement does not vary. These results underline the role of edge physics linked to the density in both $\mathrm{L}$ and $\mathrm{H}-$

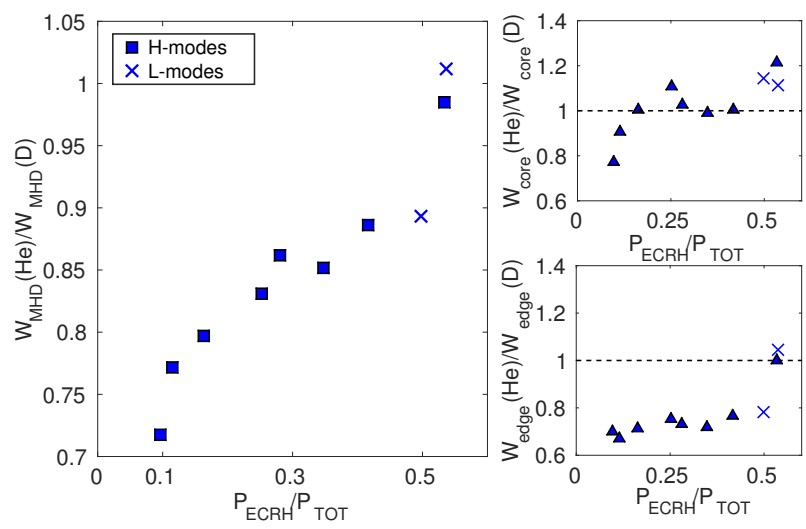

FIG. 2: Ratio of the helium to deuterium measured total plasma stored energy against the ratio of ECRH over the total heating power (left). The same comparison (right) is done by looking separatly at the core and edge contributions to the stored energies computed from the ion and electron kinetic profiles and defined in the text.

modes in the degradation of confinement of He plasmas. In this respect, the impact of thermal coupling between the ion and electron channels at the plasma edge will be discussed hereafter. Finally, we note that the lower edge confinement in He can be compensated by the core (except at low density where both core and edge confinements are higher/similar in He compared to D) yielding a strong variation of the total stored energy with respect to $P_{\text {ECRH }} / P_{\text {TOT }}$.

In Fig. 3, temperature profiles, normalised gradients $\left(R / L_{T}=-(R / T)(\partial T / \partial r)\right.$ with $R$ the major radius $)$ and the heat diffusivities $\chi$ in He and $\mathrm{D}$ are plotted for the highest and lowest stored energy ratios shown in Fig. 2 and denoted by 'ECRH' and 'NBI' respectively. These plasmas have similar electron density profiles among D and He pairs which makes the temperature profiles comparison equivalent to the kinetic stored energy comparison. To compensate the reduced number of ions, the helium temperature should be two times larger than in D. In practice, there is collisional thermal coupling between electrons and ions and this ideal increase in helium temperature is only obtainable for perfectly uncoupled electron and ion heat channels and low ion stiffness.

When confinement in He is similar to D (ECRH case with low density), not only the ion temperature in $\mathrm{He}$ is higher but also the electron temperature $T_{e}$. The increase in $T_{i}$ in He being lower than two times $T_{i}$ in $\mathrm{D}$, the gap in the ion kinetic energy is filled by the increase in the electron kinetic energy yielding the same total stored energies in He and D plasmas. This can be described by thermal coupling $\nu_{e i} \propto n_{e}^{2} Z\left(T_{e}-T_{i}\right) /\left(T_{e}^{3 / 2} m_{i}\right)$ and electron on-axis heating (provided heat diffusivities in He are similar to D). For the same input power deposited on the ions, the He temperature profile increases compared to D. This increase reduces the equipartion from 

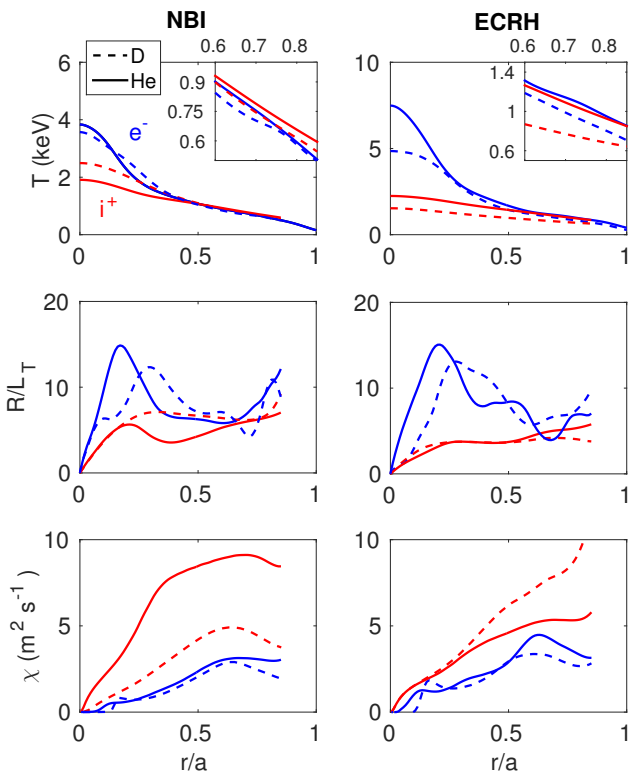

FIG. 3: Electron and ion temperature profiles for the highest NBI (left)/ECRH (right) heated plasmas. The normalised temperature gradients $R / L_{T}$ and heat diffusivities $\chi$ are also shown against $r / a$.

electrons to ions due to a decrease in $T_{e}-T_{i}$. In addition, due to dominant electron heating, the electron temperature grows further, reducing the thermal exchange and yielding a final state where both the ion and electron temperature are higher in He. This is observed in $\mathrm{L}$ and $\mathrm{H}$-modes and can be reproduced via simplified transport models. In the low density case shown here, the temperature profiles in He increase on the whole radial domain. For higher densities and dominant ECRH heating, only the core temperature profiles are increased yielding a systematic loss of confinement at the edge.

In the high NBI heating case, the ion core temperature profile is lower in He compared to D. Additionally the ion normalised temperature gradient $R / L_{T_{i}}$ is lower in the range $r / a=0.2-0.65$ and the ion heat diffusivity larger in He (more than a factor 2 for $r / a \leq 0.5$ ), showing increased turbulent transport in this case in opposition to the gyro-Bohm scaling. At the edge, whilst the ion temperature in $\mathrm{He}$ is slightly larger than that in $\mathrm{D}$, the electron temperature is identical and lower than $T_{i}$ leading to a larger thermal exchange from the ions to the electrons in He. This edge feature is also observed for all the medium/high density cases studied here (in L-modes as well). An increased stiffness in the edge electron channel could explain the limited increase of both temperature profiles in He via unfavourable thermal exchange.

Causes of reduced confinement in the core and edge of He plasmas are explored by flux-tube nonlinear and linear gyrokinetic simulations of the turbulent transport. First, electromagnetic nonlinear simulations are performed for
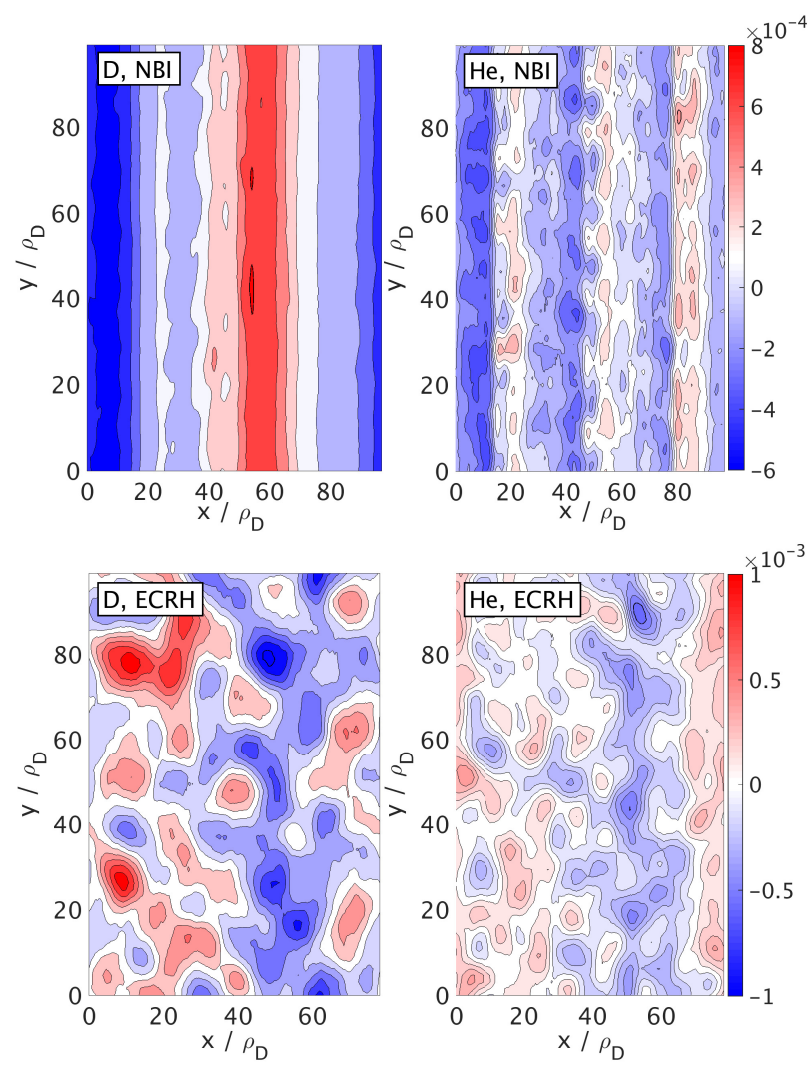

FIG. 4: Contours of the perturbed electrostatic potential in the plane perpendicular to the magnetic field at the low field side, computed from local non-linear gyrokinetic simulations of the deuterium (left panels) NBI (top) and ECRH (bottom) cases at $r / a=0.3$ and $r / a=0.5$ respectively. The same simulations have been carried out by changing only the main ion species to He (right panels). Heat fluxes for these cases are gathered in table I.

the core of the NBI and ECRH cases, at $r / a=0.3$ and $r / a=0.5$ respectively, using the gyrokinetic code GKW [14]. The gridsizes used are $n_{\mu}=10, n_{v_{\|}}=48, n_{s} / n_{p t}=$ 48 for the number of points in the magnetic moment, parallel velocity and parallel direction respectively. Box sizes in the radial $x$ and binormal $y$ directions are $90 \rho_{D}$ and $100 \rho_{D}$ with $\rho_{D}$ the deuterium Larmor radius. The Miller geometry [15] is used for the magnetic equilibrium and collisions are modelled via the pitch-angle scattering operator. For meaningful comparisons, input parameters of the D cases are used and a companion simulation is done by changing only the main ion species to He.

The normalised perturbed electrostatic potentials are compared in Fig. 4. Turbulence in the NBI case is Ion Temperature Gradient (ITG) dominated whereas in the ECRH case it is Trapped Electron Mode (TEM) dominated and related to the increased $T_{e} / T_{i}$ in the core [16]. For the ITG turbulence regime, zonal flows (in the $y$ direction) are particularly strong in D and effectively shear the turbulent vortices. In contrast, these flows are lower in the companion He simulation (convective turbulent cells are less sheared). In the TEM regime, zonal flows 
are less dominant, as also observed in previous works for high $T_{e} / T_{i}$ and low magnetic shear TEM turbulence $[17,18]$. In this case convective cells are smaller in $\mathrm{He}$, in qualitative agreement with gyro-Bohm scaling.

The role of zonal flows is further highlighted in table I where the ratio of the zonal flow perturbed electrostatic potential amplitude at $k_{y}=0$ over the sum on all non-zero toroidal modes $\left(\left|\phi_{k_{y}=0}\right|^{2} / \sum_{k_{y} \neq 0}\left|\phi_{k_{y}}\right|^{2}\right.$ and afterwards denoted by $\left.\left|\phi_{\mathrm{ZF}}\right|^{2} /\left|\phi_{\text {turb }}\right|^{2}\right)$ is shown together with the ion and electron heat fluxes for the NBI and ECRH cases from Fig. 4. These fluxes have to be compared to the experimental fluxes for the NBI case in D $Q_{i}=0.1 \mathrm{MW} / \mathrm{m}^{2}, Q_{e}=0.06 \mathrm{MW} / \mathrm{m}^{2}$ and the ECRH case $Q_{i}=0.06 \mathrm{MW} / \mathrm{m}^{2}, Q_{e}=0.09 \mathrm{MW} / \mathrm{m}^{2}$.

\begin{tabular}{lccc}
\hline \hline & $\left|\phi_{\mathrm{ZF}}\right|^{2} /\left|\phi_{\text {turb }}\right|^{2}$ & $Q_{i}$ & $Q_{e}$ \\
\hline NBI $(\mathrm{D}, \mathrm{EM})$ & $\mathbf{1 5 . 4}$ & $\mathbf{0 . 1 3}$ & $\mathbf{0 . 1}$ \\
\hline NBI $(\mathrm{D}, \mathrm{ES})$ & 3.1 & 0.94 & 0.54 \\
\hline NBI $(\mathrm{D}, \mathrm{ES}, \nu=0)$ & 1.39 & 1.2 & 0.76 \\
\hline NBI $(\mathrm{D} \rightarrow \mathrm{He}, \mathrm{EM})$ & $\mathbf{1 . 2 3}$ & $\mathbf{0 . 2 9}$ & $\mathbf{0 . 4}$ \\
\hline NBI $(\mathrm{D} \rightarrow \mathrm{He}, \mathrm{ES})$ & 1.03 & 0.35 & 0.33 \\
\hline NBI $(\mathrm{D} \rightarrow \mathrm{He}, \mathrm{ES}, \nu=0)$ & 1.65 & 0.45 & 0.72 \\
\hline ECRH $(\mathrm{D}, \mathrm{EM})$ & $\mathbf{1 . 7}$ & $\mathbf{0 . 2 5}$ & $\mathbf{0 . 7 1}$ \\
\hline ECRH $(\mathrm{D} \rightarrow \mathrm{He}, \mathrm{EM})$ & $\mathbf{0 . 9}$ & $\mathbf{0 . 0 7}$ & $\mathbf{0 . 4 1}$ \\
\hline
\end{tabular}

TABLE I: Ratio of the zonal flow strength over the turbulent strength represented by $\left|\phi_{\mathrm{ZF}}\right|^{2} /\left|\phi_{\text {turb }}\right|^{2}$ for the NBI and ECRH cases. Computed ion and electron heat fluxes in $\mathrm{MW} / \mathrm{m}^{-2}$ are also shown. Cases of Fig. 4 are written in bold.

The NBI case has been extensively studied with simulations including or not electromagnetic and collisional effects. It is shown that $\left|\phi_{\mathrm{ZF}}\right|^{2} /\left|\phi_{\text {turb }}\right|^{2}$ is particularly strong in the electromagnetic deuterium case where the heat fluxes are close to experimental levels. Electromagnetic effects strongly stabilise the turbulence while not changing the zonal flow level, thus increasing $\left|\phi_{\mathrm{ZF}}\right|^{2} /\left|\phi_{\text {turb }}\right|^{2}$. Collisions also have an additional stabilising impact. Changing the species to helium, the ratio of zonal flow to turbulence strengths is much lower than in $\mathrm{D}$ and is not particularly affected by electromagnetic effects nor collisions. More interestingly, the ion and electron heat fluxes are stronger in He compared to D in the electromagnetic case, in complete opposition to simple gyro-Bohm expectations. In the ECRH heated plasmas where TEM turbulence develops with high $T_{e} / T_{i}$ and low magnetic shear, the ratio $\left|\phi_{\mathrm{ZF}}\right|^{2} /\left|\phi_{\text {turb }}\right|^{2}$ is low (compared to the NBI D case) and the ion and electron heat fluxes are much lower in He than in D (though the computed electron heat fluxes are overestimated compared to experimental levels). Thereby, the coupling of zonal flows and electromagnetic effects is the main component breaking the gyro-Bohm scaling of turbulent radial structures which is consistent with the current experimental observations of increased transport in He in the ITG regime only. Interestingly, this is also consistent with several previous works on turbulent transport and confinement

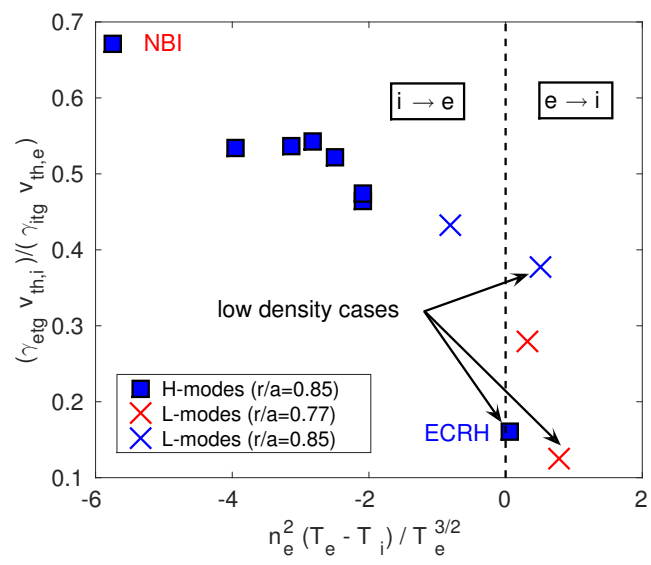

FIG. 5: Normalised ratios of the highest linear growth rates of the ETG and ITG instabilities versus the electron to ion thermal coupling strength for He plasmas.

times for D isotopes [8, 17, 19].

To account for confinement loss at the edge of He plasmas, the role of Electron Temperature Gradient (ETG) driven turbulence is investigated by linear gyrokinetic simulations. These electron scales instabilities are known to increase the electron stiffness, also via multi-scale interactions $[20,21]$ and could explain the limited increase of $T_{e}$ and $T_{i}$ compared to $\mathrm{D}$ plasmas. The ratio of the normalised most unstable mode in the electron scales denoted by $\gamma_{\text {etg }}$ over the ion scales $\gamma_{\text {itg }}$ is shown in Fig. 5 for the He discharges at $r / a=0.85$ versus the normalised thermal exchange strength. L-modes are also included for both radial locations $r / a=0.77$ and $r / a=0.85$. We note that with the normalisation used, the linear ITG growth rate in $\mathrm{He}$ is two times larger than in $\mathrm{D}$ for the same parameters [22]. It is shown that for all medium to high density cases, thermal exchange in He goes from the ions to the electrons. Additionally, ETG are destabilised in these cases. On the other hand, at low densities ETG are less destabilised (except for the L-mode at $r / a=0.85$ but $T_{e}-T_{i}$ is positive) and an increase in both the electron and ion temperature profiles is observed. Thereby, thermal coupling and ETG destabilisation at the edge of He plasmas can explain the loss of edge confinement compared to D plasmas in both L and H-modes. This result awaits a confirmation by computationally demanding multi-scale nonlinear simulations.

In conclusion, experiments in AUG have allowed the identification of plasma regimes where the confinement of He plasmas is equal to that of corresponding $\mathrm{D}$ plasmas, characterized by large fractions of $P_{\mathrm{ECRH}} / P_{\mathrm{TOT}}$. These new results are in contrast to the common observation, obtained in plasmas with larger fractions of ion heating, where the He confinement is about $70 \%$ of that of companion D plasmas. A theoretical explaination of these contrasting observations has been identified for the first time, involving a combination of core and 
edge transport effects. At low $P_{\mathrm{ECRH}} / P_{\mathrm{TOT}}$, the core and edge contributions to the He plasma total stored energies are both lower and contribute evenly to the loss of confinement time compared to D. With increasing fractions of $P_{\mathrm{ECRH}} / P_{\mathrm{TOT}}$, the core contribution increasingly compensates the loss of plasma pressure from the edge. In the extreme case of high $P_{\mathrm{ECRH}} / P_{\mathrm{TO}}$ and low density, with separation between the electron and ion heat transport channels, the edge and core contributions to the total stored energy are both beneficial, leading to the same confinement time as in D. In the core, the zonal flows, coupled to electromagnetic effects, are shown to be the main ingredients in breaking the gyro-Bohm scaling in the ITG regime (low $P_{\mathrm{ECRH}} / P_{\mathrm{TOT}}$ ), whereas they play a minor role in TEM turbulence, with high $T_{e} / T_{i}$ and low magnetic shear (high $\left.P_{\mathrm{ECRH}} / P_{\mathrm{TOT}}\right)$. The identified role of zonal flows can be connected to the observations in $\mathrm{H}$ plasmas $\left(\rho_{H}=\rho_{H e}\right)$ and provides an important element to also obtain a consistent understanding of the isotope effects. Finally, the destabilisation of ETG instabilities at the edge of He plasmas due to increased $T_{i} / T_{e}$, together with thermal coupling from the ions to the electrons, can explain the systematic loss of confinement for He plasmas in $\mathrm{L}$ and $\mathrm{H}$-modes in the peripheral region of the plasma.

The authors would like to acknowledge fruitful discussions with E. Fable and S. Freethy.

[1] D. C. McDonald et al, Plasma Physics and Controlled Fusion 46, 519 (2004)

[2] F. Ryter, T. Pütterich, M. Reich, A. Scarabosio, E. Wolfrum, R. Fischer, M. Gemisic Adamov, N. Hicks, B. Kurzan, C. Maggi, R. Neu, V. Rohde, G. Tardini and the ASDEX Upgrade TEAM, Nuclear Fusion 49, 062003 (2009)

[3] G. Manfredi and M. Ottaviani, Physical Review Letters 79, 4190 (1997)
[4] M. Bessenrodt-Weberpal et al, Nuclear Fusion 33, 1205 (1993)

[5] P. Schneider et al, Nuclear Fusion 57, 66003 (2017)

[6] Z. Lin, S. Ethier, T. S. Hahm, and W. M. Tang, Physical Review Letters 88, 195004 (2002)

[7] R. E. Waltz, R. L. Dewar and X. Garbet, Physics of Plasmas 5, 1784 (1998)

[8] J. Garcia, T. Görler, F. Jenko and G. Giruzzi, Nuclear Fusion 57, 014007 (2017)

[9] M. Nakata, M. Nunami and H. Sugama, Physical Review Letters 118, 165002 (2017)

[10] ITER Physics Expert Groups on Confinement and Transport et al, Nuclear Fusion 39, 2175 (1999)

[11] F. Sommer, J. Stober, C. Angioni, E. Fable, M. Bernert, A. Burckhart, V. Bobkov, R. Fischer, C. Fuchs, R. M. McDermott, W. Suttrop, E. Viezzer and the ASDEX Upgrade Team, Nuclear Fusion 55, 033006 (2015)

[12] F. Sommer, J. Stober, C. Angioni, E. Fable, M. Bernert, A. Burckhart, V. Bobkov, R. Fischer, C. Fuchs, R. M. McDermott, W. Suttrop, E. Viezzer and the ASDEX Upgrade Team, Nuclear Fusion 52, 114018 (2012)

[13] A. Manini et al, Plasma Phys. Control. Fusion 46, 1723 (2004)

[14] A. G. Peeters, Y. Camenen, F. J. Casson, W. A. Hornsby, A. P. Snodin, D. Strintzi, G. Szepezi, Computer Physics Commnunications 180, 2650 (2009)

[15] R. L. Miller, M. S. Chu, J. M. Greene, Y. R. Lin-Liu and R. E. Waltz, Physics of Plasmas 5, 973 (1998)

[16] Alessandro Casati, C. Bourdelle, X. Garbet and F. Imbeaux, Physics of Plasmas 15, 042310 (2008)

[17] A. Bustos, A. Banon Navarro, T. Görler, F. Jenko, and C. Hidalgo, Physics of Plasmas 22, 012305 (2015)

[18] J. Lang, S. E. Parker, Y. Chen, Physics of Plasmas 15, 055907 (2008)

[19] Y. Xu et al, Physical Review Letters 110, 265005 (2013)

[20] N.T. Howard, C. Holland, A.E. White, M. Greenwald and J. Candy, Nuclear Fusion 56, 014004 (2016)

[21] S. Maeyama, Y. Idomura, T.-H. Watanabe, M. Nakata, M. Yagi, N. Miyato, A. Ishizawa, and M. Nunami, Physical Review Letters 114, 255002 (2015)

[22] I. Pusztai, J. Candy and P. Gohil, Physics of Plasmas 18, $122501(2011)$ 